\title{
Anti-Müllerian hormone (AMH) as a good predictor of time of menopause
}

\author{
Aleksandra Kruszyńska, Jadwiga Słowińska-Srzednicka \\ Department of Endocrinology, Centre of Postgraduate Medical Education, Bielański Hospital, Warsaw, Poland
}

\begin{abstract}
Anti-Müllerian hormone (AMH) in women is secreted by granulosa cells in late preantral and small antral follicles. AMH seems to be a very stable marker having some advantages over other biochemical and biophysical markers and is very useful in the assessment of ovarian reserve. AMH measurement may be used in cases of premature ovarian failure, including iatrogenic, due to treatment for cancer, hypogonadotropic hypogonadism, and lastly, in polycystic ovary syndrome (PCOS). It is also a very specific marker of ovarian tumors - folliculomas. According to outcomes of some studies, AMH seems to be highly predictive for the timing of menopause. There are mathematical models in which a single AMH measurement is used to predict the time of menopause even in very young women, many years before the last period.
\end{abstract}

Key words: anti-Müllerian hormone (AMH), menopause, ovarian reserve, premature ovarian failure (POF).

\section{Introduction}

In women anti-Müllerian hormone (AMH) is produced in the ovary by granulosa cells of antral follicles. Then, it is released into the follicular fluid and blood vessels. In clinical practice its levels are measured in peripheral blood.

This hormone plays a significant role in the development of reproductive organs in both sexes during the embryonic period. In an adult woman its role probably consists in the regulation of folliculogenesis, predominantly in the mechanism of inhibiting primordial follicle recruitment and decreasing the sensitivity of small antral follicles to follicle-stimulating hormone (FSH) activity. To date, the main clinical application of $\mathrm{AMH}$ determination in women has been the assessment of ovarian reserve in the diag nostics of infertility, premature ovarian failure, and hypogonadotropic hypogonadism [1]. Recently published studies highlighted the value of $\mathrm{AMH}$ determination in polycystic ovary syndrome (PCOS). Seemingly, AMH may also be a very useful predictive marker of the time of menopause.

\section{AMH throughout a woman's life}

A gradual increase in $\mathrm{AMH}$ levels is observed in girls from the first day of life, with maximum levels observed in women at around the age of 25. After puberty $\mathrm{AMH}$ is produced by granulosa cells of primary, preantral and small antral (diameter: 2-4 mm) follicles, in which the highest levels of $\mathrm{AMH}$ were reported. $\mathrm{AMH}$ expression is not found in atretic follicles. In an adult woman $\mathrm{AMH}$ levels gradually decrease until they reach values below detectable limits in postmenopausal women [1].
$\mathrm{AMH}$ is considered a stable hormone as regards its levels over a menstrual cycle and demonstrates low variability in subsequent cycles, as it is a marker of non-cyclic ovarian activity. According to studies on AMH fluctuations, the lowest levels were observed during the very early luteal phase, directly after ovulation. These findings were reported with regard to young women. In the case of older women, the range of $\mathrm{AMH}$ level fluctuation over a menstrual cycle was very low [1].

It is thought that oral contraceptives (OCP) do not influence $\mathrm{AMH}$ levels. However, some studies revealed [2] that OCPs were responsible for the reduction in $\mathrm{AMH}$ levels, and the discontinuation of contraception might result in increasing the levels. Therefore, it is suggested that $\mathrm{AMH}$ measurement may be unreliable if performed when hormonal birth control methods are used. Data regarding the influence of tobacco smoking on $\mathrm{AMH}$ levels are divergent [3]. Lower AMH levels were observed in current smokers compared to never-smokers. The influence of smoking habits on $\mathrm{AMH}$ reduction is larger in the case of daily smoking and depends on the number of cigarettes smoked. Further studies are necessary for a more accurate elucidation of the relationship between smoking and the values of ovarian reserve [4].

\section{The assessment of ovarian reserve}

In clinical practice, apart from $\mathrm{AMH}$ measurement, the assessment of ovarian reserve includes the following tests: - FSH level measurement on the $3^{\text {rd }}$ day of the cycle single FSH measurement is characterized by low reli- 
ability due to its significant intra- and inter-cycle variability. It is assumed that FSH levels $>10 \mathrm{IU} / \mathrm{l}$ indicate a reduced ovarian reserve,

- the measurement of FSH and estradiol $\left(E_{2}\right)$ levels on the $3^{\text {rd }}$ day of the cycle,

- ultrasonographic determination of the number of antral follicles (AFC) sized 2-10 mm in diameter during early follicular phase,

- the measurement of ovarian volume.

$\mathrm{AMH}$ is considered a good and reliable parameter in the assessment of ovarian reserve, the best of the above-mentioned ones [5]. Its reduced levels may indicate reduced ovarian reserve, even if the woman has regular menstrual cycles and the levels of FSH and $E_{2}$ are still normal. A good correlation has been found between AMH levels and age and also between AMH levels and the number of antral follicles. The diversity of results depending on the type of laboratory test used and the absence of a universal calculation method to facilitate the comparison of results obtained via different tests is a significant limitation associated with the use of $\mathrm{AMH}$ as a test for ovarian reserve. The interpretation of the result and the cut-off values should be individually determined for each test $[6,7]$. It is usually assumed that $\mathrm{AMH}$ values of $1 \mathrm{ng} / \mathrm{ml}$ and lower may translate into reduced ovarian reserve.

\section{AMH as a new marker of the time of menopause}

The age of 51 is the average time of menopause, defined as the last menstrual period in a woman's life. Physiological menopause may occur between 40 and 60 years of age, but the decrease in the natural fertility of women starts 10-13 years prior to menopause. It was estimated that during perimenopause the number of ovarian follicles decreases below several thousand and the progression towards menopause and onwards is characterized by a very low number of follicles - estimated to be below 1000 [1]. It is suggested that the age of menopause is highly heritable [3].

Research is conducted to find factors, including biochemical and hormonal markers, which would facilitate the determination of the period of fertility and the time of menopause in women. The determination of predictors of the period of fertility in women is gaining importance because of society ageing and later motherhood. Another problem which is tackled is better prophylaxis as regards diseases which occur significantly more commonly after menopause. These include osteoporosis, cardiovascular disorders and also hormone-related neoplasms, e.g. breast cancer and endometrial cancer. Parameters which indirectly indicate the time of menopause are the ovarian volume and the number of antral follicles, which is correlated with AMH levels.
As regards hormonal factors, the determination of FSH and inhibin $B$ was found valuable, but the predictive value was lower than $\mathrm{AMH}$, because its levels decreased earlier than in the case of the above-mentioned hormones.

Several authors reported that AMH determination was a valuable predictor of menopause [8]. It is considered that the level of $\mathrm{AMH}$ is very low, or even below detectable limits approximately 5 years before menopause [5]. According to some research [9] the time of menopause may be predicted via a mathematical model on the basis of a single $\mathrm{AMH}$ measurement and the age of the patient. The obvious value of this study is the age of study group participants (20-49 years), which enables the implementation of the model in very young women and predicts the time of menopause even several decades earlier. The authors observed an even reduction in $\mathrm{AMH}$ levels in all age groups over 6 years of observation of the study cohort. Sowers et al. [10] also demonstrated that $\mathrm{AMH}$ measurement and the use of a statistical model precisely predicted the time of menopause. However, no young women were included in their study. Freeman et al. [11] reported that the levels of $\mathrm{AMH}<0.2 \mathrm{ng} / \mathrm{ml}$ occurred on average 5.99 years prior to menopause in women aged 45-48 years and 9.94 years in women aged $35-39$. As regards $A M H$ values over $1.5 \mathrm{ng} / \mathrm{ml}$, menopause occurred on average after 6.23 years in the older group and after more than 13 years in the younger group. A prospective study by Broer et al. [12] included over 250 women who underwent tests at baseline (age 21-46) and after 11 years. It was demonstrated that the time of menopause may be precisely determined on the basis of AMH level and the patient's age.

A reliable algorithm of the time of menopause may be developed if other factors that might influence the age at menopause, e.g. cigarette smoking, ethnicity or $\mathrm{BMI}$, are included. Currently, it is known that smoking accelerates the occurrence of menopause by 3 years on average $[11,13,14]$. Moreover, AMH levels decrease at a faster rate in women who smoke [15], and 38-50-yearold women who smoke have lower AMH levels than their non-smoking peers [16].

\section{AMH as a marker in premature ovarian failure}

Premature ovarian failure (POF) may be regarded as premature menopause. It is defined as the loss of ovarian function (the termination of its reproductive and hormonal function due to the depletion of ovarian follicles) before 40 years of age. POF occurs in 1/100 women before 40 years of age and in 1/1000 women before 30 . It is associated with certain chromosomal aberrations, fragile $\mathrm{X}$ syndrome, galactosemia, and FSH receptor disorders. However, the etiology most commonly remains unknown. The markers of ovarian reserve assessment are used in the diagnostics of POF. AMH level determi- 
nation is of importance here, as it is markedly lower in women with POF than in healthy ones. $\mathrm{AMH}$ measurement is highly important in the case of POF suspicion, because this parameter is characterized by low susceptibility to hormonal treatment including oral contraceptives and hormone replacement therapy. Moreover, transient or permanent character of amenorrhea in POF patients also translates into various $\mathrm{AMH}$ levels. Skałba et al. [17] observed very low or undetectable AMH levels in POF patients: $0.65 \pm 1.81 \mathrm{pmol} / \mathrm{l}(0.09 \pm 0.25 \mathrm{ng} / \mathrm{ml})$. In the case of POF patients with secondary amenorrhea lasting over 3 years the serum AMH was very low or below detectable limits: $0.16 \pm 0.10 \mathrm{pmol} / \mathrm{l}(0.02 \pm 0.015 \mathrm{ng} /$ $\mathrm{ml})$. POF patients whose menstruation resumed had AMH levels significantly higher, but markedly lower than healthy women: $3.06 \pm 3.69 \mathrm{pmol} / \mathrm{l}(0.43 \pm 0.52 \mathrm{ng} / \mathrm{ml})$ [17]. A study conducted in Holland [18] showed that AMH was a better marker of ovarian function assessment than inhibin B or AFC in women with an increased FSH level. The values of $\mathrm{AMH}$ were below the $5^{\text {th }}$ percentile in POF patients. Normal AMH values were found in $75 \%$ of regularly menstruating women with an increased FSH level and the diagnosis of incipient ovarian failure (IOF). As regards women with oligomenorrhea and an increased FSH (referred to as transitional ovarian failure - TOF), normal values of AMH were found only in 33\%. Based on research, $\mathrm{AMH}$ is considered a highly useful clinical marker confirming the diagnosis of POF.

\section{AMH as a marker of iatrogenic ovarian failure}

The loss of oocytes and the reduction in reproductive potential, and in some cases menopause, including premature, may be associated with exposure to chemotherapy, radiotherapy of the pelvic region or an operative procedure involving the ovaries. Iatrogenic gonad failure may also be observed in girls who have undergone chemotherapy due to various neoplasms occurring in childhood. It is recommended to assess ovarian reserve in women who have undergone this type of treatment. AMH appears to be a good marker of ovarian reserve assessment also in this group of women. $\mathrm{AMH}$ measurement before and after treatment may help to assess an individual risk of premature loss of fertility and consider the implementation of methods of fertility protection. Preparations which are highly ovarotoxic include cyclophosphamide, melphalan, dacarbazine, busulfan and chlormethine. Moderate ovarotoxicity is related to the use of drugs such as cisplatin, carboplatin, and doxorubicin. Low risk of fertility disturbances occurs in the case of vincristine, methotrexate, bleomycin, mercaptopurine or vinblastine. The toxic effect on gonads depends on the dose and treatment duration. It is worth noting that chemotherapeutics used in the treatment of breast cancer may be ovarotoxic. It is considered that in the case of radiotherapy ovarian dysfunction may occur with doses of $>0.06 \mathrm{~Gy}$, while doses of > 8 Gy cause irreversible ovarian failure [19].

\section{AMH in ovarian tumors}

$\mathrm{AMH}$ is secreted in women only by granulosa cells of ovarian follicles, so it is a very useful marker of tumors originating from these cells. Elevated AMH levels are reported in $76-93 \%$ of women with granulosa cell tumors (folliculoma). The levels of AMH may be markedly elevated: average levels are $190 \mathrm{ng} / \mathrm{ml}$ (ranging from 2 to $1124 \mathrm{ng} / \mathrm{ml}$ ). The elevation of $\mathrm{AMH}$ levels may precede a clinically overt tumor even by 16 months. AMH appears to be a more specific marker for these tumors than inhibin and estradiol, and the values of $\mathrm{AMH}$ correlate with tumor size. $\mathrm{AMH}$ is also a very sensitive and specific marker which facilitates early diagnosis of a recurrence in patients with folliculoma who have undergone ovariectomy. It is important in terms of a high risk of relapse even 10-20 years after the resection of the primary tumor [20].

\section{Conclusions}

The determination of the predictors of the time of menopause and developing a model which would facilitate the precise prediction of the time of menopause in a particular woman is still a challenge. The determination of AMH levels appears to be a good and reliable parameter which may accelerate the development of such a model. A single AMH measurement is more valuable in the assessment of ovarian reserve than measuring estradiol, FSH or inhibin B. Further research is necessary, including population studies, which will help to specify reference values, standardize $\mathrm{AMH}$ measurement and develop a reliable model to calculate the time of menopause. However, it seems that AMH measurement will be the most important element in this model. Its value arises from the usefulness in women at different ages (very young or perimenopausal), and it may be an effective marker in the determination of the time of menopause even many years before its occurrence.

\section{Disclosure}

Authors report no conflict of interest.

\section{References}

1. Ledger WL. Clinical utility of measurement of anti-mullerian hormone in reproductive endocrinology. J Clin Endocrinol Metab 2010, 95: 5144-5154.

2. Bentzen JG, Forman JL, Pinborg A, et al. Ovarian reserve parameters: a comparison between users and non-users of hormonal contraception. Reprod Biomed Online 2012; 25: 612-619.

3. Dewailly D, Andersen C, Balen A, et al. The physiology and clinical utility of anti-Mullerian hormone in women. Hum Reprod Update 2014; 20 : 370-385. 
4. Dólleman M, Verschuren WM, Eijkemans MJ, et al. Reproductive and lifestyle determinants of anti-Müllerian hormone in a large populationbased study. J Clin Endocrinol Metab 2013; 98: 2106-2115.

5. Broer SL, Broekmans FJ, Laven JS, et al. Anti-Müllerian hormone: ovarian reserve testing and its potential clinical implications. Hum Reprod Update 2014; 20: 688-701.

6. Committee on Gynecologic Practice. Committee opinion no. 618: Ovarian reserve testing. Obstet Gynecol 2015; 125: 268-273.

7. Practice Committee of the American Society for Reproductive Medicine. Testing and interpreting measures of ovarian reserve: a committee opinion. Fertil Steril 2012; 98: 1407-1415.

8. Tehrani FR, Solaymani-Dodaran M, Azizi F. A single test of antimullerian hormone in late reproductive-aged women is a good predictor of menopause. Menopause 2009; 16: 797-802.

9. Tehrani FR, Shakeri N, Solaymani-Dodaran M, et al. Predicting age at menopause from serum antimullerian hormone concentration. Menopause 2012; 18: 766-770.

10. Sowers MR, Eyvazzadeh AD, McConnell D, et al. Antimullerian hormone and inhibin $B$ in the definition of ovarian aging and the menopause transition. J Clin Endocrinol Metab 2008; 93: 3478-3483.

11. Freeman EW, Sammel MD, Lin H, et al. Anti-Müllerian hormone as a predictor of time to menopause in late reproductive age women. J Clin Endocrinol Metab 2012; 97: 1673-1680.

12. Broer SL, Eijkemans MJC, Scheffer GJ, et al. Anti-mullerian hormone predicts menopause: a long-term follow-up study in normoovulatory women. J Clin Endocrinol Metab 2011; 96: 2532-2539.

13. van Asselt KM, Kok HS, van Der Schouw YT, et al. Current smoking at menopause rather than duration determines the onset of natural menopause. Epidemiology 2004; 15: 634-639.

14. Fleming LE, Levis S, LeBlancWG, et al. Earlier age at menopause, work, and tobacco smoke exposure. Menopause 2008; 15: 1103-1108.

15. Sowers MR, McConnell D, Yosef M, et al. Relating smoking, obesity, insulin resistance, and ovarian biomarker changes to the final menstrual period. Ann NY Acad Sci 2010; 1204: 95-103.

16. Plante BJ, Cooper GS, Baird DD, et al. The impact of smoking on antimullerian hormone levels in women aged 38 to 50 years. Menopause 2010; 17: 571-576.

17. Skałba P, Cygal A. Anti-Müllerian hormone: plasma levels in women with polycystic ovary syndrome and with premature ovarian failure. Prz Menopauz 2011; 3: 232-236.

18. Knauff EA, Eijkemans MJ, Lambalk CB, et al. Anti-Müllerian hormone, inhibin $\mathrm{B}$, and antral follicle count in young women with ovarian failure. J Clin Endocrinol Metab 2009; 94: 786-792.

19. Dębski R. Niepłodność. In: Wielka Interna. Endokrynologia część I. Tom 12. Antczak A, Myśliwiec M, Pruszczyk P (eds.). Medical Tribune, Warszawa 2011; 581-592.

20. Karkanaki A, Vosnakis C, Panidis D. The clinical significance of anti-mullerian hormone evaluation in gynecological endocrinology. Hormones 2011; 19: 95-103. 